\title{
Actividad física y riesgo de cáncer de mama en mujeres mexicanas
}

\author{
Sandra Patricia Ortiz-Rodríguez, MSc,, (I) Gabriela Torres-Mejía, MD, PhD, (I) \\ Fernando Mainero-Ratchelous, MD, (2) Angélica Ángeles-Llerenas, MSc, ${ }^{(1)}$ Alma Ethelia López-Caudana, MSc, ${ }^{(3)}$ \\ Eduardo Lazcano-Ponce, MD, Dr en Sc, (I) Isabelle Romieu, MD, Dr en Sc. ${ }^{(1,4)}$
}

Ortiz-Rodríguez SP,Torres-Mejía G, Mainero-Ratchelous F, Ángeles-Llerenas $\mathrm{A}$, López-Caudana $\mathrm{AE}$, Lazcano-Ponce E, Romieu I. Actividad física y riesgo de cáncer de mama en mujeres mexicanas. Salud Pulica Mex 2008;50:I26-I35.

\section{Resumen}

Objetivo. Evaluar el efecto de la actividad física moderada (en horas por semana y METs-hora por semana) sobre el riesgo de cáncer de mama (CM). Material y métodos. Se trata de la primera fase de un estudio multicéntrico de casos y controles con base poblacional que se desarrolló en el Distrito Federal, Monterrey y Veracruz, México, en el año 2004. Se analizaron 58 casos y 58 controles pareados a los casos por quinquenio de edad, y pertenencia al sistema de salud; participaron tres hospitales del Instituto Mexicano del Seguro Social, tres del Instituto de Seguridad Social al Servicio de los Trabajadores del Estado y tres de la Secretaría de Salud. Resultados. En mujeres posmenopáusicas se observó una reducción del riesgo de $\mathrm{CM}$ por cada hora semanal adicional de actividad física moderada ( $R M=0.9$ I; IC95\% 0.85-0.97); en mujeres premenopáusicas, la disminución del riesgo no tuvo significancia estadística $(R M=0.99$; IC95\% 0.94-I.05) $(p=0.048$, modificación de efecto). Conclusiones. La actividad física reduce el riesgo de CM en mujeres mexicanas posmenopáusicas.

Palabras clave: actividad física moderada; cáncer de mama; México
Ortiz-Rodríguez SP,Torres-Mejía G, Mainero-Ratchelous F, Ángeles-Llerenas $\mathrm{A}$, López-Caudana $\mathrm{AE}$,

Lazcano-Ponce E, Romieu I.

Physical activity and breast cancer

risk in Mexican women.

Salud Pulica Mex 2008;50:126-135.

\section{Abstract}

Objective. To evaluate the effect of moderate physical activity (hours per week and METs hours per week) on the risk of breast cancer (BC) in Mexican women. Material and Methods. This is the initial stage of a case control multicentric study based in the Federal District, Monterrey and Veracruz, Mexico, during 2004. Fifty eight cases paired to 58 control cases on quinquennium of age, and belonging to the health system were analyzed: three hospitals from the IMSS three from ISSSTE and three from SS participated. Results. In postmenopausal women, there was a reduction of the risk in BC by every additional hour per week of moderate physical activity $(R M=0.91 ; / C 95 \%$ 0.85-0.97); in premenopausal women, the reduction of the risk was not statistically significant $(R M=0.99 ;$ IC95\% 0.94-I.05) ( $p=0.048$, effect modification). Conclusions. Moderate physical activity reduces the risk of $B C$ in postmenopausal Mexican women.

Key words: moderate physical activity; breast cancer risk; Mexico

(I) Centro de Investigación en Salud Poblacional, Instituto Nacional de Salud Pública. Cuernavaca, Morelos, México

(2) Hospital de Gineco-Obstetricia No. 4, IMSS. Ciudad de México

(3) Hospital General Regional Licenciado Ignacio García Téllez, IMSS. Cuernavaca, Morelos

(4) The National Center for Environmental Health-Centers for Disease Control and Prevention.Atlanta GA, EUA

Fecha de recibido: 7 de febrero de 2007 - Fecha de aceptado: 4 de octubre de 2007

Solicitud de sobretiros: Dra. Gabriela Torres Mejía. Instituto Nacional de Salud Pública. Av. Universidad 655

Col. Santa María Ahuacatitlán. 62508 Cuernavaca, Morelos, México.

Correo electrónico:gtorres@insp.mx 
R esultados de diversos estudios observacionales sugieren que la actividad física vigorosa reduce el riesgo de padecer cáncer de mama (CM); sin embargo, el papel de la actividad física moderada es aún incierto. Desde el punto de vista de la salud pública, la actividad física es un factor modificable; de ahí la importancia de estudiar su relación con el riesgo de CM. ${ }^{1}$ Existen diversas hipótesis sobre los mecanismos biológicos plausibles que explican el vínculo entre actividad física moderada y riesgo de $\mathrm{CM}$ que incluyen cambios en el metabolismo de las hormonas sexuales, ${ }^{2}$ resistencia a la insulina y factores de crecimiento, disminución de la obesidad y posibles cambios en la función inmunitaria. ${ }^{3}$ Se ha observado que la reducción del riesgo difiere por el estado de la menopausia debido a que existe mayor evidencia de tal reducción en los estudios realizados en mujeres posmenopáusicas. ${ }^{4}$ En las mujeres premenopáusicas se sugiere que la obesidad disminuye el riesgo de CM por la leptina (hormona producida por los adipocitos), la cual inhibe la producción de estrógenos ováricos, que tienen un efecto mitógeno en las células del tejido mamario. Se sabe que la cantidad de mutaciones irreparables en el ADN depende de la tasa de división celular. ${ }^{5}$ Por el contrario, en las mujeres posmenopáusicas la obesidad viene aparejada a un incremento en el riesgo de CM debido, en parte, a que el tejido adiposo es el sitio de mayor producción de estrógenos y a que existe una disminución en los valores libres de la hormona ligadora de hormonas sexuales (SHBG, del inglés sex hormona binding globulin), con el consecuente aumento de la biodisponibilidad de estrógenos. Esto explica, de manera parcial, por qué el efecto de la actividad física difiere entre mujeres pre y posmenopáusicas. ${ }^{4,6} \mathrm{~A}$ pesar de las diferencias en los mecanismos biológicos que afectan la reducción en el riesgo de $\mathrm{CM}$, pocos estudios han evaluado en la misma población, con semejante diseño y métodos, el efecto de la actividad física moderada y de la actividad física total en mujeres pre y posmenopáusicas. ${ }^{1,4}$

A causa de la tendencia creciente a reconocer el valor de la actividad física de intensidad moderada para la prevención de enfermedades crónicas por parte de instancias como el Departamento de Salud y Servicios Humanos de los Estados Unidos, ${ }^{7}$ y a que hasta donde se sabe en la población mexicana no se ha evaluado dicho vínculo, el objetivo del presente estudio fue estimar la relación de la actividad física moderada y la actividad física total con el riesgo de $\mathrm{CM}$ en mujeres que participaron en la primera fase de la investigación "Factores de riesgo de CM en México, un estudio multicéntrico".

\section{Material y métodos \\ Diseño y población de estudio}

En el año 2004 se realizó un estudio de casos y controles en Monterrey, Veracruz y el Distrito Federal. Este trabajo fue aprobado por los comités de ética de las instituciones participantes, y las mujeres que intervinieron firmaron una carta de consentimiento informado. Los controles fueron poblacionales para tres de las sedes, y hospitalarios para cinco de ellas; los controles se parearon con los casos por quinquenio de edad y por hospital. Con el propósito de lograr el número de participantes requeridas para esta primera fase, se invitó a participar a un total de 100 casos y 100 controles. Se incluyó a 10\% de los casos y controles del estudio multicéntrico con el objeto de valorar las condiciones operativas del estudio definitivo. Participaron nueve hospitales de Ginecología y Oncología ubicados en las ciudades de Monterrey, Veracruz y el Distrito Federal. Tres de los hospitales están adscritos al Instituto Mexicano del Seguro Social (IMSS); tres, al Instituto de Seguridad Social al Servicio de los Trabajadores del Estado (ISSSTE), y tres, a la Secretaría de Salud (SS).

Los casos elegibles fueron mujeres entre 35 y 69 años de edad con CM incidente diagnosticado por medios histopatológicos, in situ o invasivo, de origen primario y con residencia, durante los últimos cinco años, en el Distrito Federal o área conurbada, Monterrey o Veracruz. Asimismo, fue requisito que las pacientes no hubieran recibido tratamiento para tumores como radioterapia, quimioterapia o antiestrogénicos en el último año, ni tomado antiestrogénicos por otras razones en los seis meses recientes y que no estuvieran embarazadas ni lactando.

Se incluyeron casos incidentes que se diagnosticaron de manera consecutiva desde el inicio del estudio y que cumplieron con los criterios de inclusión. Sin embargo, para establecer la distribución por edad de los casos se incorporaron con base en la distribución nacional de la edad reportada en el Registro Histopatológico de Neoplasias Malignas de México del año 2002. ${ }^{8}$

Además de los criterios descritos para los casos en los controles se incluyó el de considerar mujeres aparentemente sanas. Los controles para el Distrito Federal se seleccionaron mediante un muestreo probabilístico complejo multietápico.

Etapa I: se realizó una selección probabilística de delegaciones con un número suficiente de mujeres dere- 
chohabientes del IMSS, ISSSTE y no afiliadas (SS). Las delegaciones escogidas fueron Magdalena Contreras, Gustavo A. Madero y Coyoacán.

Etapa II: selección probabilística de cinco Áreas Geoestadísticas Básicas (AGEBs) que garantizaron un número suficiente de mujeres de 35 a 69 años de edad.

Etapa III: se elaboró un marco muestral vivienda por vivienda para identificar a las mujeres que cumplían con los criterios de inclusión. Con este listado se realizó la selección aleatoria de los controles, de tal forma que la muestra correspondiera a la distribución por edad establecida para los casos. Por cuestiones logísticas, la selección de los controles para los estados de Nuevo León y Veracruz se realizó a partir de las acompañantes de pacientes que se encontraban en los servicios de Consulta Externa de cada uno de los hospitales.

\section{Recolección de la información}

En una entrevista realizada por personal capacitado y estandarizado, las mujeres del estudio proporcionaron información sobre su salud, dieta y actividad física; se les realizaron mediciones antropométricas y se obtuvieron muestras sanguíneas. Mediante el cuestionario de salud se recolectaron datos acerca de sus características sociodemográficas, reproductivas y de antecedentes patológicos. Para valorar la dieta en el año previo a la fecha del estudio se aplicó el cuestionario semicuantitativo de frecuencia de consumo de alimentos elaborado por Willet y colaboradores, el cual se adaptó para la población mexicana y se validó en la población de la Ciudad de México. ${ }^{9}$

El nivel socioeconómico se midió a través del análisis de componentes principales, que se hizo con las variables de características de las viviendas y tenencia de electrodomésticos. Las variables relacionadas con la tenencia de electrodomésticos incluyó sólo las preguntas: ¿En su casa tienen computadora? y ¿En su casa tienen coche?

Para medir la actividad física, se revisaron los cuestionarios Seven-Day Physical Activity Recall, ${ }^{10}$ a los que se les efectuó una modificación y adaptación a la población mexicana ya que se desarrolló un estudio piloto. Se obtuvo información acerca del tiempo utilizado en llevar a cabo diversas actividades durante una semana típica que reflejara la actividad desarrollada la mayor parte del tiempo durante los últimos 12 meses. Para determinar el tipo de actividad física, se tuvo en cuenta el compendio propuesto por Ainsworth y colaboradores, ${ }^{11}$ que clasifica cada una de las actividades de acuerdo con su intensidad. La intensidad de la actividad física se mide en METs (equivalentes metabólicos), en que un MET se define como el gasto de energía que un adulto promedio gasta en sentarse tranquilamente y que es de alrededor de una kilocaloría por kilogramo de peso corporal por hora. Así, la intensidad de las actividades se estableció por la tasa de gasto de energía propuesta por Ainsworth y colaboradores ${ }^{11}$ expresada en METs, y se clasificaron como sigue: $a$ ) sueño; $b$ ) actividades ligeras (i.e. aquellas que requieren esfuerzo físico leve o en las que casi no hay actividad y que tienen un consumo de energía de 1.0 a $3.4 \mathrm{METs}$, e.g conducir un coche, labores de oficina, leer un libro y ver televisión); c) actividades moderadas (i.e. aquellas que producen cansancio físico pero no quitan el aliento y que tienen un consumo de energía de 3.5-5.9 METs; e.g. lavar el coche, pintar muros, empacar y desempacar bolsas, yoga, ciclismo ligero y caminata ligera), y d) actividades vigorosas (i.e. aquellas que hacen sudar, aumentan la frecuencia cardiaca o quitan el aliento, y que tienen un consumo de energía mayor o igual a 6 METs, e.g. subir escaleras con bolsas pesadas, mover muebles, correr, trabajo de granja). Las participantes indicaron el número de horas que utilizaban al día para realizar cada una de las actividades durante una semana.

Para cuantificar la actividad física total se multiplicaron las horas semanales de actividad física ligera por 2.2 METs; las de actividad física moderada por 4.7 $M E T s$, y las de actividad física vigorosa por 6 METs. Los valores de METs empleados corresponden al gasto promedio de energía de acuerdo con la intensidad de cada actividad. Por último, se sumaron los METs de los tres tipos de actividad.

Se obtuvieron mediciones antropométricas de todas las participantes (i.e. talla, peso, circunferencia de cintura y cadera) con base en los procedimientos recomendados durante la encuesta NHANES III (del inglés National Health and Nutrition Examination Survey). El peso se midió con una báscula mecánica digital modelo 1631 con capacidad máxima de $150 \mathrm{~kg}$ (TANITA Corporation, Tokio, Japón), y la altura con un estadímetro de dos metros con unidades divididas en milímetros (Seca bodymeter 280, Corporation, Tokio, Japón).

\section{Análisis estadístico}

Con el propósito de describir a la población de estudio según sus características sociodemográficas, historia médica y medidas antropométricas se utilizaron medidas de frecuencia, de tendencia central y de dispersión, de acuerdo con las características de la distribución de cada una de las variables.

Se compararon las distribuciones de las características de interés entre los grupos de casos y controles de acuerdo con la condición de la menopausia, así como en la muestra total, mediante el uso de pruebas estadísticas 
deji cuadrada y de Wilcoxon para variables cualitativas y ordinales, respectivamente. El efecto de la actividad física moderada y de la actividad física total (METs-horas por semana) sobre el riesgo de CM se estimó mediante razones de momios $(R M)$, a partir de modelos de regresión logística condicional simple. Se realizaron modelos bivariados y multivariados, y se ajustó por cada una de las variables de interés. Para seleccionar el modelo que mejor explicaba los datos, se corrió un modelo de regresión logística condicional saturado que incluía las variables potencialmente confusoras, a partir del cual las covariables con menor significancia se eliminaron poco a poco para observar los cambios en el efecto principal. Se mantuvieron en el modelo final las covariables que afectaban en más de $10 \%$ la magnitud de los estimadores, así como aquellas que en estudios previos mostraron ser potencialmente confusoras. La modificación del efecto de la actividad física en el riesgo de CM por condición de menopausia se evaluó mediante la comparación del logaritmo natural de la verosimilitud de los modelos de regresión logística múltiple condicional con y sin término de interacción. El modelo final propuesto se valoró mediante el análisis de puntos influyentes y la estimación de residuos de desvianza, y se sometió a pruebas de bondad de ajuste. ${ }^{12}$ Se estimaron coeficientes de correlación de Spearman entre el número de horas de actividad física moderada reportados en dos momentos diferentes en una submuestra del estudio. El análisis estadístico se realizó con los programas Stata versión 9.0 y SPSS versión 14 .

\section{Evaluación de la reproducibilidad del cuestionario de actividad física}

Se llevó a cabo un estudio de reproducibilidad interobservador mediante la aplicación del cuestionario de actividad física en una muestra preliminar de 38 casos consecutivos que se diagnosticaron entre abril y julio de 2006. El intervalo de tiempo promedio entre la primera y la segunda aplicación fue de un mes. Estos casos se tomaron del estudio definitivo y los aplicaron dos enfermeras con capacitación previa. La reproducibilidad del cuestionario se determinó mediante el cálculo de coeficientes de correlación de Spearman entre la primera y la segunda medición para cada tipo de actividad física.

\section{Resultados}

\section{Características de la población de estudio}

De los 100 casos y 100 controles invitados al estudio, 85 casos y 71 controles accedieron a participar. Con el objeto de analizar un control por cada caso pareado por quinquenio de edad y por hospital se consideraron en el análisis 58 casos y 58 controles. No se observaron diferencias significativas en términos estadísticos entre las mujeres incluidas y excluidas del análisis para las variables de actividad física moderada, edad de la menarca, paridad, edad de la madre al primer embarazo, duración de la lactancia, edad de la menopausia, índice de masa corporal y consumo de calorías (cuadro I). De la población analizada, $50 \%$ de las mujeres era derechohabiente del IMSS, 12\% del ISSSTE y 28.4\% manifestó no contar con seguridad social. El perfil de los casos correspondió a mujeres de 51 años, casadas, amas de casa, de estrato socioeconómico bajo y con escolaridad de secundaria o nivel técnico. Los controles tuvieron el mismo perfil, a excepción de tener escolaridad primaria. Más de 95\% de las mujeres que tomaron parte en el estudio carecía de antecedentes de $\mathrm{CM}$ en algún familiar de primer grado. La menarca se presentó con mayor frecuencia entre los

Cuadro I

DifERENCIAS ENTRE LOS CASOS Y CONTROLES INCLUIDOS Y EXCLUIDOS DEL ANÁLISIS POR CARACTERíSTICAS GENERALES de la población de estudio. Ciudad de México, Monterrey y Veracruz, 2005

\begin{tabular}{|c|c|c|c|c|c|c|c|c|c|}
\hline \multirow[b]{3}{*}{ Actividad física moderada (horas semana) } & \multicolumn{4}{|c|}{ Casos } & \multicolumn{5}{|c|}{ Controles } \\
\hline & \multirow[b]{2}{*}{7} & \multirow{2}{*}{$\begin{array}{l}\text { Incluidos* } \\
(0-30)\end{array}$} & \multirow{2}{*}{$\begin{array}{l}\text { Excluidos* } \\
10.5(0.5-21.0)\end{array}$} & \multirow{2}{*}{$\begin{array}{c}p^{\ddagger} \\
0.31\end{array}$} & \multicolumn{2}{|c|}{ Incluidos* } & \multicolumn{2}{|c|}{ Excluidos* } & \multirow{2}{*}{$\begin{array}{c}p^{\ddagger} \\
0.22\end{array}$} \\
\hline & & & & & 13.5 & $(6.0-26.5)$ & 28 & $(8-37)$ & \\
\hline Edad de la menarca (años) & 13 & $(12-14)$ & $12(12-14)$ & 0.78 & 13 & $(11-14)$ & II & $(11-13)$ & 0.05 \\
\hline Paridad (número de partos) & 3 & $(2-4)$ & $(1-4)$ & 0.45 & 3 & $(2-4)$ & 3 & $(2-3)$ & 0.60 \\
\hline Edad de la madre al primer embarazo a término (años) & & $(18-26)$ & $25 \quad(21-29)$ & 0.27 & 23 & $(19-26)$ & 22 & $(17-25)$ & 0.38 \\
\hline Duración total de la lactancia ( meses) & 11.5 & $(3-3 I)$ & $12 \quad(0.5-36)$ & 0.97 & 14 & $(6-29)$ & 15 & $(14-46)$ & 0.25 \\
\hline Edad de la menopausia (años) & & $(43-5 I)$ & $47 \quad(4 \mid-48)$ & 0.07 & 46 & $(42-50)$ & 41 & $(40-44)$ & 0.12 \\
\hline Consumo diario de calorías & 2046.2 & $(1599.3-258 \mid .7)$ & $1997.3(|632.7-3| \mid 3.3)$ & 0.55 & 2187.3 & 36.7-2599.5) & 2444.7 & $79.2-3 \mid 42.8)$ & 0.22 \\
\hline \multicolumn{10}{|l|}{ * Mediana (rango intercuartil) } \\
\hline
\end{tabular}


12 y 13 años de edad. Aproximadamente la tercera parte de las mujeres era multigesta, refirió antecedente de uso de hormonales orales y presentó la menopausia antes de los 45 años, con antecedente de uso de terapia hormonal de reemplazo en $15 \%$ de las mujeres posmenopáusicas; $56 \%$ con antecedentes de lactar por un tiempo mayor de un año; $85 \%$ de los casos y $95 \%$ de los controles tuvieron su primer hijo antes de los 30 años de edad; $15 \%$ refirió consumo de alcohol en el año anterior al estudio, y $90 \%$ de los casos y $85 \%$ de los controles se encontraban con sobrepeso u obesidad, con una mediana de consumo energético de 2137 kilocalorías por día. La comparación entre casos y controles por condición de menopausia sólo mostró diferencias estadísticas significativas con el primer embarazo a término $(p=0.01)$, y con sobrepeso u obesidad $(p=0.04)$ en las mujeres posmenopáusicas (cuadro II).

No se encontraron diferencias estadísticas significativas entre el total de casos y de controles respecto del número de horas semanales de actividad física ligera e intensa, mientras que sí se detectaron para el número de horas semanales de actividad física moderada $(p<0.001)$ (cuadro III). Al interior de los grupos de mujeres pre y posmenopáusicas únicamente se observaron diferencias estadísticamente significativas en las horas de actividad física moderada entre los casos y los controles del grupo de mujeres posmenopáusicas $(p<0.01)$ (cuadro III).

Cuando la actividad física se midió en METs, las disparidades fueron irrelevantes entre los casos y los controles en las mujeres pre y posmenopáusicas.

En el cuadro IV se muestra el análisis bivariado de los factores que se relacionaron con $\mathrm{CM}$.

\section{Relación entre actividad física y CM}

En el análisis multivariado se observó significancia estadística para el término interacción entre horas de actividad física moderada y estado de menopausia ( $p=0.048$ ), lo que representó un efecto protector de este tipo de actividad para $\mathrm{CM}$ en mujeres posmenopáusicas $(R M=0.91$; IC95\% 0.85-0.97) y una ausencia de efecto de la actividad física moderada sobre el CM en mujeres premenopáusicas ( $R M=0.99$; IC95\% 0.94-1.05), después de ajustar por estrato socioeconómico, paridad, tiempo de uso de anticonceptivos, consumo de alcohol en el último año, IMC y consumo diario de calorías (cuadro V).

Además, en las mujeres posmenopáusicas, por cada 10 METs/horas semanales de actividad física total, se observó una reducción del riesgo de significancia estadística ( $R M=0.88$; IC95\% 0.79-0.99); sin embargo, el efecto en las mujeres premenopáusicas careció de relevancia estadística $(R M=0.95$; IC95\% 0.84-1.09). (cuadro V).

\section{Resultados preliminares del estudio de reproducibilidad interobservador del cuestionario de actividad física}

Distintas enfermeras aplicaron el cuestionario en dos ocasiones a 38 mujeres que tenían diagnóstico de $\mathrm{CM}$ con una tasa de respuesta de $95 \%$. El promedio de edad de las encuestadas fue de $58.8 \pm 9.7$ años. Treinta y seis de ellas (94.8\%) sabían leer, y de éstas, 38\% había terminado la educación básica primaria, mientras que 21\% contaba con estudios técnicos. Del universo de mujeres, $71 \%$ era ama de casa; $10.5 \%$, estilista o administradora; $7.9 \%$ hacía ropa o alimentos para vender y $10.7 \%$ era empleada, obrera o comerciante; $42.1 \%$ sufría de obesidad $\left(\right.$ IMC $>30 \mathrm{~kg} / \mathrm{m}^{2}$ ), 31.6\% tenía sobrepeso (IMC 25-29.9 $\mathrm{kg} / \mathrm{m}^{2}$ ) y sólo $26.2 \%$ poseía un índice de masa corporal normal (IMC $\left.<25 \mathrm{~kg} / \mathrm{m}^{2}\right)$. El coeficiente de correlación de Spearman fue de 0.69 para horas de actividad física ligera $(p<0.001)$, de 0.67 para horas de sueño $(p<0.001)$, de 0.40 para horas de actividad física moderada $(p=0.01)$ y de 0.31 para actividad física vigorosa $(p=0.05)$.

\section{Discusión}

En este estudio se encontró una reducción del riesgo de enfermar en el rango de 8 a 33\%, similar al que otros investigadores comunican. ${ }^{1,14-17}$ En parte, esta variación se debe al uso de métodos diferentes, a los tipos de actividad física y a los periodos de la vida evaluados. En el presente estudio se observó que la actividad física moderada es un factor protector de $\mathrm{CM}$ en las mujeres posmenopáusicas pero no en las premenopáusicas; estos resultados son consistentes con estudios previos de casos y controles, ${ }^{1,13}$ de cohorte ${ }^{14-16}$ y con el informe de una revisión sistemática. ${ }^{17}$ Sin embargo, el hallazgo debe interpretarse con cautela debido al reducido número de mujeres premenopáusicas que abarcó este estudio. Tal vez la actividad física reciente haya ocasionado la reducción de peso en estas mujeres y, por tanto, su posibilidad de desarrollar $\mathrm{CM}$.

El presente estudio también documentó una modificación del efecto de la actividad física por condición de menopausia sobre el riesgo de CM. Este resultado es consistente con estudios publicados de manera previa en el sentido de que la actividad física ejerce mayor efecto sobre el CM en las mujeres posmenopáusicas. Otro estudio reciente mostró mayor influencia de esta misma exposición antes $(R M=0.67$; IC $0.46-0.96)$ y después (0.70 IC95\% 0.59-0.94) de la menopausia. ${ }^{1}$ No obstante, es prudente esperar que los hallazgos se confirmen, luego de que se termine de desarrollar la propuesta original. De cualquier forma, es conveniente considerar que uno de los principales motivos que se se- 


\section{Cuadro II}

\section{Características SOCIOdemográficas, Reproductivas y ANTROPOMÉtricas EN LA POBLACIÓN DE ESTUDIO y por condición de menopausia en Ciudad de México, Monterrey y Veracruz. 2005}

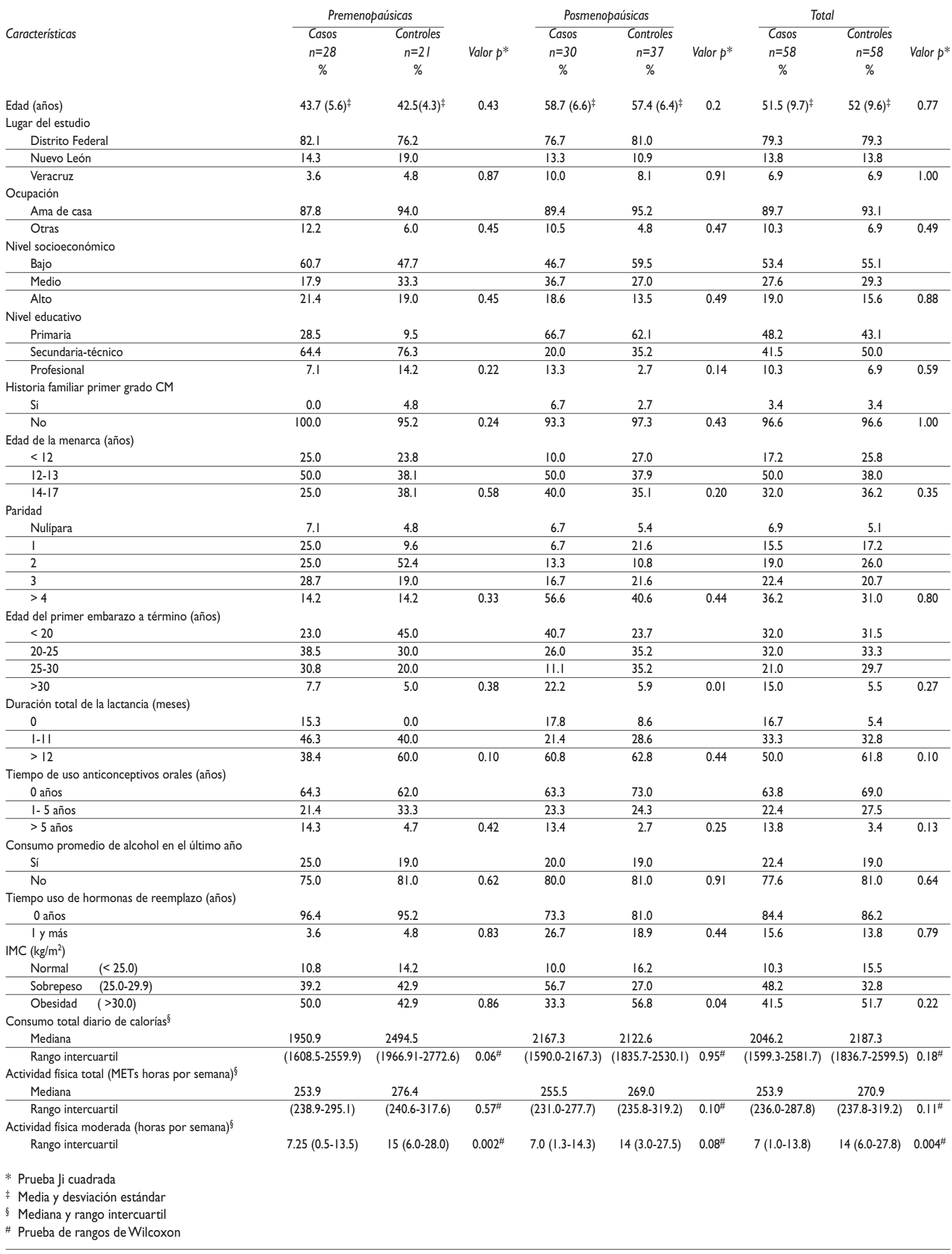




\section{Cuadro III}

Mediana de tipos actividad física de acuerdo con su inTensidad (EQUivalentes metabólicos hora SEMana) EN LA POBLACIÓN DE ESTUDIO Y POR CONDICIÓN DE MENOPAUSIA EN MUJERES de la Ciudad de México, Monterrey y Veracruz. 2005

\begin{tabular}{|c|c|c|c|c|c|c|c|c|c|}
\hline \multirow{4}{*}{ Características } & \multicolumn{2}{|c|}{ Premenopaúsicas } & \multicolumn{3}{|c|}{ Posmenopaúsicas } & & \multicolumn{2}{|c|}{ Total } & \multirow{4}{*}{ Valor $p^{\ddagger}$} \\
\hline & Casos* & Controles* & \multirow{3}{*}{ Valor $p^{\ddagger}$} & Casos* & Controles* & \multirow{3}{*}{ Valor $p^{\ddagger}$} & Casos* & Controles* & \\
\hline & $n=28$ & $n=21$ & & $n=30$ & $n=37$ & & $n=58$ & $n=58$ & \\
\hline & $\%$ & $\%$ & & $\%$ & $\%$ & & $\%$ & $\%$ & \\
\hline \multicolumn{10}{|c|}{ Tipo de actividad física } \\
\hline Ligera & $95.0(86.75-105.25)$ & $95.5(85.0-98.5)$ & 0.55 & $92.7(83.5-104.5)$ & $89(76.0-96.5)$ & 0.06 & $93.2(84.0-105.5)$ & $91.5(76.5-98.0)$ & 0.06 \\
\hline Moderada & $7.0(1.25-15.5)$ & $12(3.0-20.0)$ & 0.20 & $7.25(0.5-11.0)$ & $16(6.0-30.0)$ & 0.003 & $7(1.0-14.0)$ & I3.5 (6.0-26.5) & 0.001 \\
\hline Intensa & $-\S$ & $-\S$ & & $-\S$ & $-\#$ & & $-\S$ & $-\#$ & \\
\hline $\begin{array}{l}\text { * Mediana y ra } \\
\text { ‡ Prueba de ra } \\
\S \text { Se observar } \\
\text { \# Se observar }\end{array}$ & $\begin{array}{l}\text { cuartil de horas sema } \\
\text { Vilcoxon } \\
\text { de actividad física inte } \\
\text { e actividad física inte }\end{array}$ & $\begin{array}{l}\text { nales } \\
\text { isa a partir de la } \\
\text { isa a partir de la }\end{array}$ & $\begin{array}{l}\text { percen } \\
\text { percen }\end{array}$ & $\begin{array}{l}\text { ila } 75 \% \\
\text { ila } 90 \%\end{array}$ & & & & & \\
\hline
\end{tabular}

ñalan para documentar falsas modificaciones de efecto consiste en la medición errónea de la exposición, factor que en este estudio se minimizó al realizar un estudio piloto que permitió documentar la confiabilidad de las mediciones.

La literatura más reciente sugiere varios mecanismos que explican la plausibilidad biológica de la reducción en el riesgo encontrada en este estudio.,3,18-19 Llama la atención que la actividad vigorosa que comparte mecanismos similares no tenga el mismo efecto en cuanto a la reducción del riesgo. Esto se puede atribuir a la baja proporción de mujeres con actividad física vigorosa en el presente estudio (28.4\%).

En cuanto a la actividad física ligera, los resultados son congruentes con los señalados en un estudio de mujeres mexicanas de 20 a 80 años de edad, en donde se notificó que la proporción de mujeres inactivas era de $30.8 \% .{ }^{20}$ En contraste, en el presente trabajo se encontró que $35.3 \%$ de las mujeres sólo realizó actividad física ligera, que es equivalente a ser inactivas. Quizá la diferencia se deba a que en aquel estudio ${ }^{20}$ sólo se evaluó actividad recreativa y en el presente se incorporaron todos los tipos de actividad física y, de este modo, se estimó el tiempo dedicado a realizar actividades recreativas, ocupacionales y del hogar. Esta última medición es de suma importancia para la presente población porque se resolvió que, en comparación con las mujeres blancas estadounidenses, las mujeres latinas dedican un promedio de tiempo mayor a actividades relacionadas con el hogar que a actividades recreativas. ${ }^{1}$ Esto ayudó a reducir el error de mala clasificación de la exposición en las participantes, ya que si se evalúa sólo la actividad recreativa se podría clasificar de manera errónea a una mujer activa como sedentaria. Además, se cuantificaron tiempo, frecuencia, intensidad y tipo de actividad física. Cabe subrayar que pocos estudios han evaluado la actividad física de manera integral. ${ }^{13}$

Cuando se valoró la actividad física total medida en METs, se encontró una reducción del riesgo en las mujeres posmenopáusicas pero no en las premenopáusicas, aunque la modificación del efecto no arrojó datos estadísticos significativos $(p=0.41)$. Estos resultados pueden reflejar un problema de poder, ya que los METs son una medida más aproximada al gasto de energía que el número de horas semanales. Esta investigación contempló un ajuste por variables potencialmente confusoras. Además de las indicadas en los modelos múltiples finales, se realizó un ajuste por antecedente familiar de CM, edad de la menarca, edad del primer embarazo a término, duración total de la lactancia y actividad física vigorosa; sin embargo, al ajustar por estas variables no se observaron cambios importantes en el efecto de interés (datos no presentados).

En este trabajo se analizaron los datos preliminares del estudio de reproducibilidad interobservador del cuestionario utilizado para medir la actividad física mediante la evaluación del grado de concordancia de los resultados al obtenerse en más de una ocasión. ${ }^{21}$ Por tal motivo es necesario valorar de modo más específico los factores que pudieron influir en la variabilidad de las respuestas.

Pocos estudios han evaluado el efecto de la actividad física sobre el CM en la población hispana. ${ }^{22,23}$ Hasta donde se sabe, el presente trabajo está entre los primeros que se realizan en población hispana y es el primero en mexicanas. El porcentaje de mujeres que realizó cuando menos media hora diaria de actividad física moderada fue de $71.5 \%$, y de actividad vigorosa, $17.2 \%$, lo cual sig- 


\section{Cuadro IV}

FACTORES ASOCIADOS A CÁNCER DE MAMA EN LA POBLACIÓN DE ESTUdIO Y POR CONDICIÓN DE MENOPAUSIA. Ciudad de México, Monterrey y Veracruz. 2005

Características

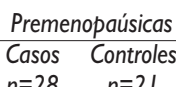

RM (IC95\%)*

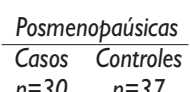

$\frac{\text { Total }}{\text { Casos Controles }}$

$R M(I C 95 \%)$

RM (IC95\%)

Nivel socioeconómico

\begin{tabular}{|c|c|c|c|c|c|c|c|c|c|}
\hline Bajo & 17 & 10 & 1.0 & 14 & 22 & 1.0 & 31 & 32 & 1.0 \\
\hline Medio & 5 & 7 & $0.37(0.07-1.76)$ & II & 10 & $1.48(0.46-4.75)$ & 16 & 17 & $0.97(0.4 I-2.33)$ \\
\hline Alto & 6 & 4 & $0.49(0.08-2.76)$ & 5 & 5 & I.38 (0.22-8.60) & II & 9 & $1.30(0.44-3.86)$ \\
\hline
\end{tabular}

Edad de la menarca (años)

\begin{tabular}{lrrrrrrrrrrr}
$<12$ & 7 & 5 & 1.0 & 3 & 10 & 1.0 & 10 & 15 & 1.0 & 10 & 14 \\
\hline $12-13$ & 14 & 8 & $0.49(0.08-2.87)$ & 15 & 14 & $2.27(0.45-11.43)$ & 29 & 22 & $1.33(0.42-4.13)$ \\
\hline $14-17$ & 7 & 8 & $0.72(0.13-3.85)$ & 12 & 13 & $3.22(0.77-13.37)$ & 19 & 21 & $2.07(2.75-5.76)$
\end{tabular}

Edad del primer embarazo a término (años)

\begin{tabular}{|c|c|c|c|c|c|c|c|c|c|}
\hline$<20$ & 6 & 10 & 1.0 & 12 & 9 & 1.0 & 18 & 19 & 1.0 \\
\hline $20-25$ & II & 6 & $2.9(0.54-16.3)$ & 8 & 13 & $0.30(0.05-1.76)$ & 19 & 19 & I.08 (0.38-3.08) \\
\hline $25-30$ & 9 & 4 & $5.45(0.79-37.16)$ & 3 & 13 & $0.07(0.00-0.66)$ & 12 & 17 & $0.70(0.22-2.22)$ \\
\hline$>30$ & 2 & I & $4.04(0.29-54.72)$ & 7 & 2 & $0.67(0.08-5.19)$ & 9 & 3 & $2.05(0.45-9.35)$ \\
\hline
\end{tabular}

Tiempo de uso de anticonceptivos orales

\begin{tabular}{lrrrrrrrrrr}
0 años & 18 & 13 & 1.0 & 19 & 27 & 1.0 & 12 & 1.0 & 37 & 12 \\
\hline I- 5 años & 6 & 7 & $0.56(0.13-2.38)$ & 7 & 9 & $1.23(0.40-3.79)$ & 13 & 22 & $0.92(0.39-2.17)$ \\
\hline$>5$ años & 4 & 1 & $1.90(0.18-19.68)$ & 4 & 1 & $6.12(0.61-61.12)$ & 8 & 3 & $4.03(0.81-19.85)$
\end{tabular}

Consumo promedio de alcohol en el último año

\begin{tabular}{|c|c|c|c|c|c|c|c|c|c|}
\hline Sí & 7 & 14 & 1.0 & 6 & 7 & 1.0 & 13 & II & 1.0 \\
\hline No & 21 & 7 & $0.78(0.18-3.26)$ & 24 & 30 & $0.8 \mathrm{I}(0.25-2.59)$ & 45 & 47 & $0.81(0.33-1.97)$ \\
\hline
\end{tabular}

IMC $\left(\mathrm{kg} / \mathrm{m}^{2}\right)$

\begin{tabular}{|c|c|c|c|c|c|c|c|c|c|c|}
\hline Normal & $(<25.0)$ & 3 & 3 & 1.0 & 3 & 6 & 1.0 & 6 & 6 & 1.0 \\
\hline Sobrepeso & $(25.0-29.9)$ & II & 9 & I.55 (0.23-10.22) & 17 & 10 & $3.46(0.66-18.10)$ & 28 & 19 & 2.II $(0.65-6.80)$ \\
\hline Obesidad & $(>30.0)$ & 14 & 9 & I.7I (0.25-II.6I) & 10 & 21 & $1.39(0.28-6.89)$ & 24 & 30 & $\mathrm{I} .2 \mathrm{I}(0.38-3.8 \mathrm{I})$ \\
\hline
\end{tabular}

Edad de la menopausia (años)

\begin{tabular}{|c|c|c|c|c|c|c|c|c|c|}
\hline$<44$ & - & - & - & 8 & 12 & 1.0 & 8 & 12 & 1.0 \\
\hline $45-54$ & - & - & - & 20 & 22 & $1.22(0.37-3.99)$ & 20 & 22 & $1.22(0.37-3.99)$ \\
\hline$>55$ & - & - & - & 2 & 3 & $0.72(0.09-5.49)$ & 2 & 3 & $0.72(0.09-5.49)$ \\
\hline
\end{tabular}
* RM= Razones de momios; $\mathrm{IC}=$ Intervalo de confianza; análisis de regresión logística simple condicional, ajustado en el diseño por quinquenio de edad y por
hospital

Las variables que a continuación se mencionan no se incluyeron en el análisis por ausencia de valores en alguna de las celdas: historia familiar de cáncer de mama, paridad, duración total de lactancia y tiempo de uso de hormonas de reemplazo

nifica que la mayoría de las mujeres del estudio cumple con las recomendaciones actuales de actividad física para mantener la salud. ${ }^{7}$ Este resultado es relevante comparado con el de mujeres inglesas, ${ }^{24}$ en donde se informa que $24 \%$ de las mujeres realiza actividad física moderada, y 5\% actividad física vigorosa, y cuyas tasas de CM son mayores que en el caso de las mexicanas.
Llama la atención que, según el estudio mencionado, a pesar de que la mayoría de las mujeres realiza por lo menos media hora diaria de actividad física moderada, $42.3 \%$ son obesas. Esto quizá se deba a que la actividad física moderada que realizan no sea efectiva para reducir el peso sino para mantenerlo, o que influyan otros factores como predisposición genética a la obesidad o una 
CuadroV

\section{CONDICIÓN DE LA MENOPAUSIA COMO MODIFICADORA \\ DE LA ACTIVIDAD FÍSICA SOBRE EL RIESGO DE CÁNCER de mama en mujeres mexicanas de la CiUdad de México, Monterrey y Veracruz. 2005}

\begin{tabular}{|c|c|c|c|c|}
\hline Características & $\begin{array}{l}\text { Casos } \\
n=58\end{array}$ & $\begin{array}{c}\text { Controles } \\
n=58\end{array}$ & $R M^{*}$ & $1 C 95 \%$ \\
\hline \multicolumn{5}{|l|}{ Actividad física moderada (horas-semana) } \\
\hline \multicolumn{5}{|l|}{ Condición de la menopausia } \\
\hline Premenopáusicas & 30 & 37 & 0.99 & $0.94-1.05$ \\
\hline Posmenopáusicas & 28 & 31 & 0.91 & $0.85-0.97$ \\
\hline Término de interacción $n^{\ddagger}$ (valor p) & & & & 0.048 \\
\hline \multicolumn{5}{|l|}{ Actividad física total§ (METs/horas-semana) } \\
\hline \multicolumn{5}{|l|}{ Condición de la menopausia } \\
\hline Premenopáusicas & 30 & 37 & 0.95 & $0.84-1.09$ \\
\hline Posmenopáusicas & 28 & 31 & 0.88 & $0.79-0.99$ \\
\hline Término de interacción ${ }^{\#}$ (valor p) & & & & 0.413 \\
\hline \multicolumn{5}{|c|}{$\begin{array}{l}\text { * RM ajustada por edad y hospital de atención en el diseño, así como por } \\
\text { estrato socioeconómico, paridad, tiempo de uso de anticonceptivos, } \\
\text { consumo promedio de alcohol en el último año, IMCy consumo de calorías } \\
\text { en el análisis } \\
\text { ‡ Interacción entre condición de la menopausia y actividad física } \\
\text { moderada } \\
\S \text { Incluye actividad física ligera, moderada y vigorosa. Se calculó la RM por } \\
\text { cada incremento de } 10 \text { METs/horas-semana } \\
\text { \# Interacción entre condición de la menopausia y actividad física total }\end{array}$} \\
\hline
\end{tabular}

dieta que las haga subir de peso no obstante la actividad física moderada. Esto es congruente con el hallazgo de que sólo $17.2 \%$ de la población realiza actividad física vigorosa, más vinculada con la reducción de peso.

Es posible que en el presente trabajo se haya introducido un error de mala clasificación no diferencial que pudo resultar en una subestimación del efecto. En 1997 Ainsworth y colaboradores realizaron un metaanálisis de evaluación de los métodos para medir la actividad física; allí se encontró que sólo 1 de 20 estudios midió la validez y reproducibilidad del cuestionario utilizado. ${ }^{25}$ En esta investigación, los valores del coeficiente de correlación de Spearman para actividad física moderada indicaron una correlación moderada que podría considerarse como una debilidad del estudio si reflejara errores de medición por parte de las entrevistadoras; sin embargo, la magnitud del coeficiente también puede relacionarse con el número de mujeres entrevistadas.

Se identifican algunas limitaciones en el trabajo; por ejemplo, el hecho de que el muestreo complejo puede ocasionar que exista una correlación entre las observaciones de un mismo conglomerado, que lleva a subestimar las varianzas de los datos y produce que los intervalos de confianza sean más estrechos y que los niveles de significancia sean mayores. Otra limitación corresponde a la distinta forma de selección de los controles en dos de las tres ciudades participantes, lo cual debe modificarse en la fase final de este estudio. La principal limitación de los controles hospitalarios es la posibilidad de que la exposición se vincule con la enfermedad o con el motivo de la consulta.

El efecto de la actividad física sobre el riesgo de $\mathrm{CM}$ pudo atribuirse a la condición de ser caso; sin embargo, al incluir casos incidentes disminuye esta posibilidad. Asimismo, se midió la actividad física realizada en el último año, y puede argumentarse que la ventana crítica para el desarrollo de cáncer no corresponde al año previo a su detección. No obstante, se acepta que la modificación de estilos de vida suele ocurrir en lapsos de tiempo mayores de un año. Es necesario realizar estudios longitudinales para evaluar el efecto de la actividad física en diferentes etapas de la vida.

En este estudio, ni las pacientes ni las enfermeras conocían la hipótesis de que la actividad física moderada reduce el riesgo de $\mathrm{CM}$, por lo que se considera que es muy reducida la probabilidad de ocurrencia de sesgo del observador o de memoria (recall bias) que habría resultado en una sobreestimación del efecto de la exposición.

En fechas recientes se ha propuesto que valores séricos altos de insulina e IGF-I tendrían utilidad como predictores intermedios para el CM. ${ }^{26}$ Por tal motivo y porque en el estudio definitivo se medirán concentraciones de este factor, se recomienda evaluar no sólo su papel como marcador intermedio para $\mathrm{CM}$ sino su relación con la actividad física moderada para contribuir al conocimiento de los mecanismos hormonales que explican el efecto de la actividad física sobre el CM.

Los datos sugieren que las mujeres del estudio pueden reducir de manera importante el riesgo de $\mathrm{CM}$ mediante la realización de actividad física moderada. De igual forma se estableció que la mujer mexicana lleva a cabo más actividad moderada por actividades del hogar y del trabajo que por actividades recreativas.

Los resultados de la investigación mostraron que la actividad física moderada disminuye el riesgo de $\mathrm{CM}$ en mujeres posmenopáusicas. Aunque los resultados deben tomarse con cautela debido al reducido número de casos y controles y a la limitada representatividad, todos éstos son consistentes con la literatura epidemiológica sobre el tema. Por lo anterior, se sugiere implementar políticas públicas orientadas a estimular la práctica de la actividad física en el ámbito familiar, laboral y comunitario. 


\section{Agradecimientos}

Agradecemos al CONACyT por el apoyo económico brindado para la realización de este trabajo. A los maestros Salvador Zamora y Aarón Salinas por sus comentarios a la presente investigación. Asimismo, a todos los médicos responsables del proyecto en los diferentes hospitales participantes: Dr. Germán Castelazo, Dr. Sinhué Barroso Bravo, Dr. Joaquín Zarco Méndez, Dr. Hernando Miranda Hernández, Dr. Edelmiro Pérez Rodríguez, Dr. Jesús Pablo Esparza Cano, Dr. Heriberto Fabela, Dr. Luis G. Menchaca Ramos, Dr. Fausto Hernández Morales, Dr. Vicente A. Saldaña Quiroz y Dra. Celina Valle.

\section{Referencias}

I. John EM, Horn-Ross P, Koo J. Lifetime physical activity and breast cancer risk in a multiethnic population:The San Francisco Bay area breast cancer study. Cancer Epidemiol Biomarkers Prev 2003; I2: | | 43- | | 52.

2. Bernstein L, Ross RK, Lobo R, Hanisch R, Krailo M, Henderson B.

The effects of moderate physical activity on menstrual cycle patterns in adolescence: implications for breast cancer prevention. $\mathrm{Br} J$ Cancer 1985; 74: 74I-745.

3. International Agency for Research on Cancer. Weight control and physical activity. Handbooks of Cancer Prevention. Metabolic consequences of overweight, underweight and physical activity/inactivity. Lyon, France: IARC Press, 2002;(6):53-82.

4. Friedenreich CM. Physical activity and breast cancer risk: the effect of menopausal status. Exerc Sport Sci Rev 2004;32:180-184.

5. Kaaks R, Lukanova A. Effects of weight control and physical activity in cancer prevention: role of endogenous hormone metabolism. Ann NY Acad Sci 2002; 963: 268-28I.

6. Friedenreich CM, Orestein MR. Physical and cancer prevention: etiologic evidence and biological mechanisms. J Nutric 2002; 132:3456S-3464S. 7. United States Department of Health and Human Services. Physical activity and health: a report of the surgeon general.Atlanta, GA: US Department of Health and Human Services, Centers for Disease Control and Prevention, National Center for Chronic Disease Prevention and Health Promotion, The President's Council of Physical Fitness and Sports, 1996.

8. Dirección General de Epidemiología de México, Dirección de Vigilancia Epidemiológica y Enfermedades No Transmisibles. Ciudad de México: Registro Histopatológico de Neoplasias Malignas (RHNM) 2002. 9. Hernández-Avila M, Romieu I, Parra S, Hernández-Avila J, Madrigal H, Willett W.Validity and reproducibility of a food frequency questionnaire to assess dietary intake of women living in Mexico City. Salud Publica Mex 1998; 40: 133-140.

10. Seven-Day Physical Activity Recall. A Collection of Physical Activity Questionnaires for Health-Related Research. Med Sci Sport Exerc 1997;29(Suppl 6):S89-SI03.

I I. Ainsworth BE, Haskell WL, Whitt MC, Irwin L, Swartz A, Strath S et al. Compendium of physical activities: an update of activity codes and MET intensities. Med Sci Sports Exerc 2000; 32: S498-5I6.

12. Schlesselman J], Stolley PD. Case - control studies. Design, conduct, analysis. En: Basic Methods of Analisis. New York: Oxford University Pres, 1982:170-290.

13. Friedenreich CM, Courneya K, Briant S. Relation between intensity of physical activity and breast cancer risk reduction. Med Sci Sports Exerc 200I; 33: I538-1545.

I4. Verloop J, Rookus MA, van der Kooy K, van Leeuwen FE. Physical activity and breast cancer risk in women aged 20-54 years. J Natl Cancer Inst 2000; 92: 128-135.

I5. Breslow R, Ballard-Barbash R, Muñoz K, Graudard B. Long-term recreational physical activity and breast cancer in the National Health and Nutrition Examination Survey I Epidemiologic Follow-Up Study. Cancer Epidemiol Biomarkers Prev 200I; 10: 805-808.

16. Moradi T, Hans-Olov A,Anders E. Physical activity and risk for breast cancer: a prospective cohort study among Swedish twins. Int J Cancer 2002; 100: 76-81.

17. Thune I, Furberg A. Physical activity and cancer risk: dose-response and cancer, all sites and site- specific. Med Sci Sports Exerc 2000: S530-S550. 18. Harlow SD, Matanoski, GM. The association between weight, physical activity, and stress and variation in the length of the menstrual cycle.Am J Epidemiol 199|; |33: 38-49.

19. Cauley JA, Gutai JP, Kuller LH, Ledone D, Powell G. The epidemiology of serum sex hormones in posmenopausal women. Am J Epidemiol 1989; 129: || $20-|| 3 \mid$.

20. López AE,Téllez M, Hernández M, Juárez S, Lazcano E, Salmerón J et al. Predictors of bone mineral density in female workers in Morelos State, Mexico.Arch Med Res 2004; 35: 172-180.

21. Szklo M, Nieto J. Epidemiología intermedia. Conceptos y aplicaciones. En:Aseguramiento y control de la calidad. Ediciones Díaz de Santos; 2003. 22. Guilliland FD, Li YF, Baumgartner K, Crumly D, Samet J. Physical activity and breast cancer risk in hispanic and non-hispanic white women.Am J Epidemiol 200I;154:442-450.

23. Slattery M, Sweeney L, Edwards S, Herrick J, Murtaugh M, Baungartner $\mathrm{K}$, et al. Physical activity patterns and obesity in hispanic and non hispanic white women. Med Sci Sports Exerc 2006;38:33-4I.

24. Livingstone MBE, Robson PJ. How active are we? Levels of routine physical activity in children and adults. Proc Nutr Soc 2003;62(3):68I-70I. 25. Ainsworth BE, Sternfeld B, Slattery LM. Physical activity and breast cancer evaluation of physical activity assessment methods. Cancer Supplement 1998;83(3):61 I-620.

26. Chlebowski RT, Pettinger M, Stefanick ML, Howard BV, MossavarRahmani Y, McTiernan A. Insulin, physical activity, and caloric intake in postmenopausal women: breast cancer implications.J Clin Oncol 2004; 22: 4507-45।3. 